\title{
Effect of inflammatory marker activity after intra-articular injection of autologous platelet-rich plasma in dogs with osteoarthritis")
}

\author{
MUSTAFA ARICAN, ATILLA ŞIMŞEK*, KURTULUŞ PARLAK, \\ KAMIL ATLI*, GONCA SÖNMEZ ${ }^{*}$
}

Department of Surgery, *Department of Virology, **Department of Genetics, Faculty of Veterinary Medicine, Selçuk University, Konya, Turkey

Arican M., Şimşek A., Parlak K., Atli K., Sönmez G.

\section{Effect of inflammatory marker activity after intra-articular injection of autologous platelet-rich plasma in dogs with osteoarthritis}

Summary

Osteoarthritis (OA) is a common cause of lameness, pain, and dysfunction in dogs. To determine the levels

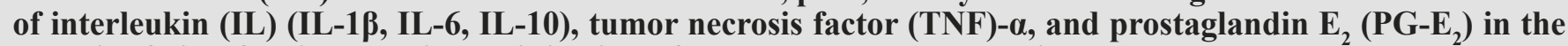
synovial fluid after intra-articular injection of an autologous platelet-rich plasma concentrate to treat dogs with osteoarthritis. Twenty dogs with osteoarthritis $(\mathrm{OA})$ were used as a materials. Fourteen dogs were used as a platelet-rich plasma (PRP) treatment group and 6 dogs were used as a control $(0.9 \% \mathrm{NaCl})$. PRP was obtained by the double centrifuge method. Affected joints were examined by radiography and ultrasonography. Lameness and pain severity were evaluated by attending clinicians. Samples were collected under sterile conditions at pre-treatment, days $1,3,5,7,15$, and weeks 4,8 , and 12 . The marker levels were determined by an enzyme-linked immunosorbent assay. No adverse effects of the injection of platelet concentrate or saline solution were observed. No significant differences were found in the Hudson Visual Analog Scale and Canine Brief Pain Inventory scores between weeks 0,4 , and 12 in the control dogs. No significant changes were observed in IL-1 $\beta$, IL-6, IL-10, TNF- $\alpha$, and PG-E ${ }_{2}$ levels $(P>0.05)$ in synovial fluid from the PRP treatment group compared to synovial fluid from the control group, although fluctuations in parameter levels were observed in both groups. Despite some variable results in inflammatory parameters, clinical improvement was recorded in the PRP-treated group. PRP injection could be an effective and safe method for treatment of dogs with osteoarthritis.

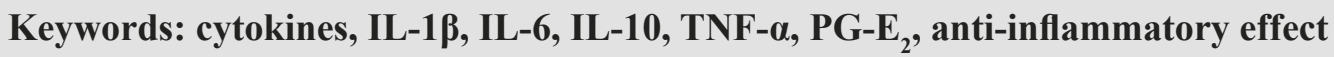

Osteoarthritis $(\mathrm{OA})$ is a common cause of lameness, pain, and dysfunction in dogs and is estimated to affect $>20 \%$ of all dogs over 1year of age (2). Cartilage breakdown products, resulting from mechanical or enzymatic destruction, induce the release of inflammatory cytokines (e.g., interleukin (IL-1, IL-6) or tumor necrosis factor $(\mathrm{TNF}-\alpha)$ ) by macrophages and chondrocytes, leading to the upregulation of matrix metalloproteinases (MMPs), nitric oxide (NO), and other proteolytic enzymes. Synovial macrophages play a role in triggering inflammatory and destructive conditions through the production of IL- $1 \beta$ and TNF- $\alpha$, which stimulate chondrocytes to produce inflammatory mediators. IL-1 $\beta$ and TNF- $\alpha$ are the main inflammatory factors involved in OA, with IL- $1 \beta$ driving tissue destruction and TNF- $\alpha$

This work was supported by The Scientific and Technological Research Council of Turkey (TÜBİTAK, Project no. 213O175). being associated with inflammation $(1,3)$. In addition, other cytokines have also been implicated, including IL-6, IL-15, IL-17, IL-18, IL-21, leukemia inhibitory factor, and IL-8 (a chemokine) $(1,4)$.

Platelet-rich plasma (PRP) is a concentrated source of autologous platelets, which contains several different growth factors (platelet-derived growth factor (PDGF), transforming growth factor-beta (TGF- $\beta 1)$, epidermal growth factor (EGF), and vascular endothelial growth factor (VEGF)) and other cytokines that can stimulate the healing of soft tissue $(8,10)$. Intra-articular injection of autologous platelets has great promise as a potential treatment for OA in dogs (9). When used in the treatment of OA, PRP is thought to reduce inflammation, slow or reverse cartilage degradation, improve blood supply, improve comfort, as well as decrease cartilage damage, synovial membrane hyperplasia, and hemorrhage 
(11). There is a relative lack of information regarding the efficacy of intra-articular PRP in the treatment of $\mathrm{OA}$ in dogs.

The aim of the present study was to determine the levels of cytokines and inflammatory mediators (IL-1 $\beta$, IL-6, IL-10, TNF- $\alpha$, and PG-E ${ }_{2}$ ) in synovial fluid samples from OA joints and to assess the efficacy of a single intra-articular injection of APC in the treatment of OA in dogs.

\section{Material and methods}

Study design. Twenty dogs (weighing 25 to $50 \mathrm{~kg}$, mean age 8.6 years) with unilateral stifle OA were used in the current study. The study was conducted as a randomized, controlled trial in the Department of Surgery, Faculty of Veterinary Medicine, University of Selcuk. The study design followed published guidelines and was approved by the institutional Animal Care and Use Committee of the Faculty of Veterinary Medicine. At the time of study enrollment, 14 dogs were assigned PRP treatment, and six dogs were used as a control group ( $0.9 \%$ saline). The dogs had clinical evidence of unilateral lameness with the cause localized to a single joint. OA was classified as primary or secondary, depending on the cause. Secondary OA was diagnosed when abnormalities causing joint instability (e.g., cranial cruciate ligament rupture) were evident. Dogs with suspected meniscal damage were excluded from the study. Only dogs with no previous treatment, including administration of nutritional supplements, were included in the study. The affected joint was examined by radiography and ultrasonography. Scores for severity of lameness and pain were assigned by an attending veterinarian in the clinic.

Radiographic examination. Affected joints were examined radiographically at pre-treatment and weeks 4, 8, 12 . In the assessment of joints, particular attention was paid to increased synovial fluid volume, displacement of the infrapatellar fat pad, enthesophyte formation, periarticular osteophyte formation, subchondral bone sclerosis, narrowing of the joint space, and mineralization of intra-articular and periarticular soft tissues. For all twenty dogs, OA was classified as mild (1), moderate (2), moderate to severe (2 to 3 ), or severe (3) as previously described (3).

Ultrasonographic examinations. Sonographic examination of full-weight-bearing dogs was performed at pretreatment and weeks 4, 8, and 12. A diagnostic ultrasound machine (Esaote Piemedical, Model 410477) with a 5-7.5 $\mathrm{MHz}$ convex transducer was used to image the joint both in sagittal and transverse planes for all the dogs.

Evaluation of lameness and pain. Lameness and pain assessments were performed at pre-treatment, days 1, 3, 5, 7, 15, and weeks 4, 8, and 12. The Hudson Visual Analog Scale (HVAS) (13) and Canine Brief Pain Inventory (CBPI) $(5,6)$ was used in assessing the scores for severity of lameness and pain. The study was managed in a blind manner. Each dog was always assessed by the same veterinarian.

Preparation and administration of autologous PRP. After these initial evaluations had been completed, dogs in the treatment group were sedated with propofol (4-7 mg/IV) and a $20 \mathrm{cc}$ blood sample was obtained from each dog by jugular vein puncture. PRP was prepared by the buffy-coat method. Five $\mathrm{ml}$ of blood was collected into each tubes in combination with Na-citrate. A double centrifuge method was used to obtain PRP. Initially, the blood was centrifuged at $96 \mathrm{~g}$ for $10 \mathrm{~min}$, and plasma was carefully removed from the centrifuge tube, followed by a second spin at $380 \mathrm{~g}$ for $20 \mathrm{~min}$. Platelets were collected from the buffy coat. All blood samples were sent to the hematology laboratory for platelet, white blood cell (WBC) and hematocrit (hct) analysis. Platelet concentrate was filtered and injected until sufficient resistance to push back the syringe plunger was reached (mean injection 3-5 $\mathrm{ml}$ depending upon the weight of the dog). For the dogs in the control group, arthrocentesis was performed as described for dogs in the treatment group, and saline solution was injected in a similar manner to the PRP.

Synovial fluid. Synovial fluid was collected under sterile conditions at pre-treatment, days $1,3,5,7,15$, and weeks 4,8 , and 12 . Although a slight contamination with blood was inevitable, severely contaminated samples (by gross appearance) were discarded and not used for the biochemical marker assays. Synovial fluid samples were subjected to routine cytological analysis and then centrifuged at $5.000 \mathrm{~g}$ for $5 \mathrm{~min}$ to remove cells and debris. The samples were stored in aliquots at $-80^{\circ} \mathrm{C}$.

ELISA. The synovial samples were aliquoted and stored at $-80^{\circ} \mathrm{C}$ until used. Double sandwich ELISA protocols were used for IL-1 $\beta$ (Cat. No: 201-15-0171), IL-6 (Cat. No: 201 -15-0128), IL-10 (Cat.No:201-15-0125), TNF- $\alpha$ (Cat. No: 201-15-0019) (Sun-Red Biological Technology Co., Ltd, Shanghai) and PG-E (Cat. No: MBS705363, My BioSource, USA). The detection ranges of the assay were $0.3-70 \mathrm{pg} / \mathrm{ml}$ for IL-1 $\beta, 0.05-15 \mathrm{pg} / \mathrm{ml}$ for IL-6, 0.5-150 ng/ml for IL-10, $0.03-9 \mathrm{pg} / \mathrm{ml}$ for TNF- $\alpha$ and $31.25-2000 \mathrm{pg} / \mathrm{ml}$ for PG-E, as reported by the manufacturers.

Statistical analyses. Using the computer-based Minitab program for the analysis of IL-1 $\beta$, IL- 6 , IL-10, TNF- $\alpha$, and PG- $\mathrm{E}_{2}$, the general linear model was used over the normal distribution values by the Anderson-Darling test. $\mathrm{P}<0.05$ was regarded as statistically significant. In addition, parametric data obtained from the control (saline-injected) and experimental (PRP treatment) group animals were evaluated as mean \pm SE. Results were interpreted by ANOVA and Tukey test (SPSS 19.0). A value of $\mathrm{P}<0.05$ was again regarded as significant.

\section{Results and discussion}

The dogs used in this study included a mix of breeds representing a variety of medium to large breeds. Body weight was $\sim 40 \mathrm{~kg}$, and age ranged from 8 to 10 years. We observed no adverse effects associated with injection of platelet concentrate or saline solution.

Radiographic OA scores at the pre-treatment examination revealed that nine dogs had moderate OA (radiographic grade 2), seven had moderate to severe OA (radiographic grade 2-3), and four had severe OA (radiographic grade 3). For all dogs, radiographic scores assigned at week 12 were the same as scores assigned at pre-treatment.

Ultrasonographic evaluations. Many cases showed capsular distension with effusion, filled with inflammatory liquid effusion and, as a result, clearly or indistinctly demarcated from the periarticular soft tissue. It was difficult to examine the articular cartilage because of the adjacent bone. Ultrasound examination of all joints (included in the study) revealed an increasing effusion 


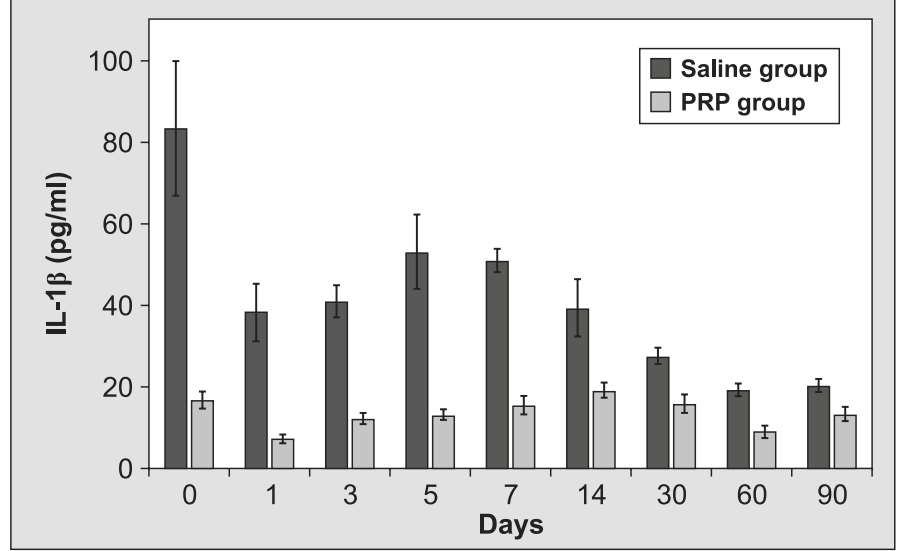

Fig. 1. Changes in IL-1 $(\mathrm{pg} / \mathrm{ml})$ levels in saline-injected group and PRP treatment group (mean $\pm \mathrm{SE}$ )

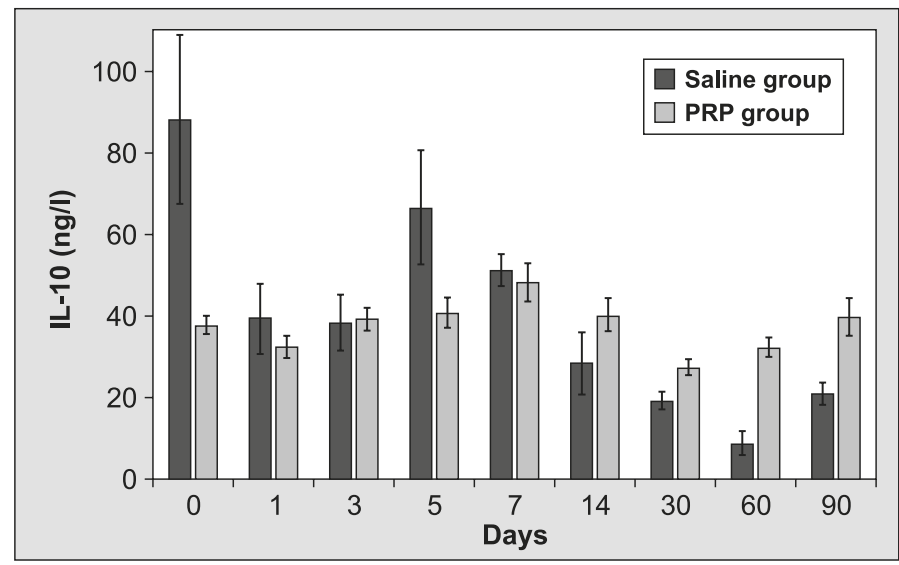

Fig. 3. Changes in IL-10 (ng/ml) levels in saline-injected group and PRP treatment group (mean $\pm \mathrm{SE}$ )

with thick and moderate homogeneous echoic structure at day 0 .

Platelet count, WBC count, and Hct. The platelet count in the concentrates (mean 1.420 .000 platelets $/ \mu \mathrm{L}$ ) was significantly higher than the platelet count in the blood samples, representing a 3- or 4-fold increase in platelet count. The WBC count $(1.09 \mathrm{mcL})$ for the platelet concentrates was significantly decreased. Hct for the platelet concentrates was significantly lower than Hct for the blood samples.

Lameness evaluation. The HVAS and CBPI scores were determined by the clinicians for all components. For the dogs in the PRP group, the HVAS components (mood, attitude, comfort, activity, playfulness, exercise, and walking comfort), and the CBPI components (pain, general activity, the ability to enjoy life, rise, walk, run, and climb) were significantly different between pretreatment and week $4(\mathrm{P}<0.05)$, week $8(\mathrm{P}<0.05)$, and week $12(\mathrm{P}<0.05)$.

ELISA results. Changes in synovial IL- $1 \beta$, IL-6, IL-10, TNF- $\alpha$, and PG-E levels in the group injected with saline $(0.9 \%)$ and in the PRP treatment group are presented in Figures 1, 2, 3, 4, and 5, respectively.

In synovial fluid from osteoarthritic dogs, IL-1 $\beta$, IL-6, IL-10, TNF- $\alpha$, and PG-E concentrations $(\mathrm{P}>0.05)$ did not undergo statistically significant changes in either the control or the PRP treatment groups, although fluctuations in all of these parameters were observed.



Fig. 2. Changes in IL-6 (pg/ml) levels in saline-injected group and PRP treatment group (mean $\pm \mathrm{SE}$ )

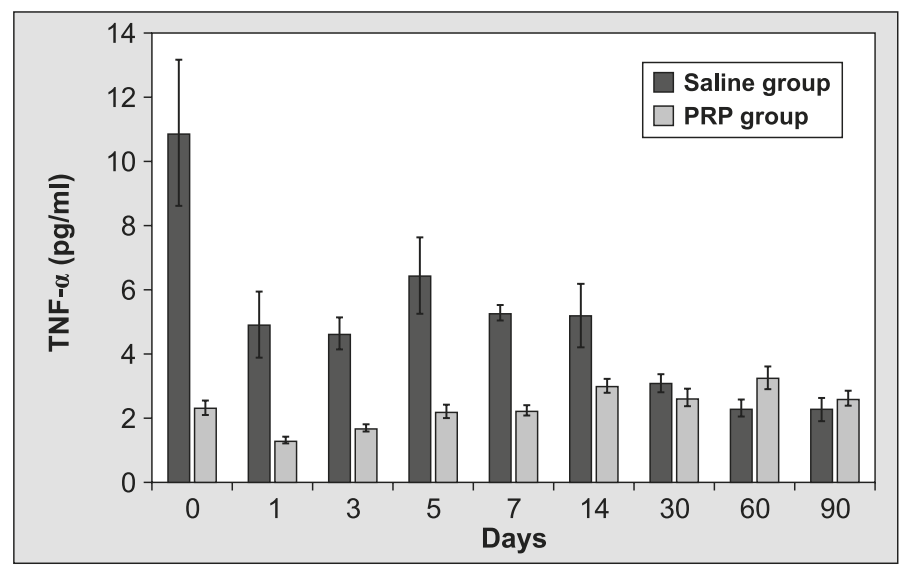

Fig. 4. Changes in TNF- $\alpha(\mathrm{pg} / \mathrm{ml})$ levels in saline-injected group and PRP treatment group (mean $\pm \mathrm{SE}$ )

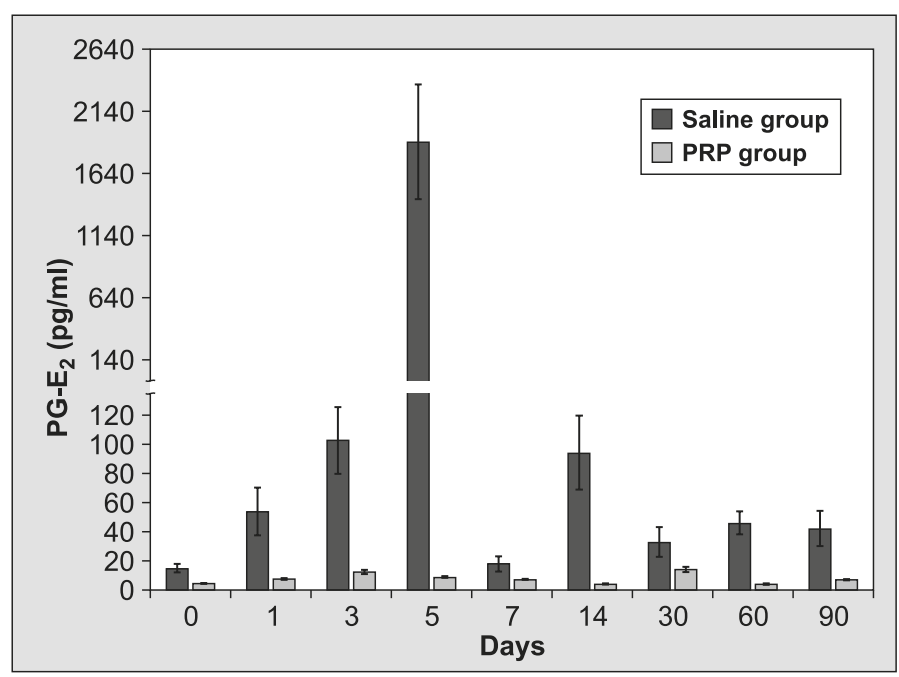

Fig. 5. Changes in PG-E $(\mathrm{pg} / \mathrm{ml})$ levels in saline-injected group and PRP treatment group (mean $\pm \mathrm{SE}$ )

IL-1 $\beta$, IL-6, IL-10, TNF- $\alpha$, and PG-E concentrations $^{2}$ in the synovial fluid from the control group are presented in Table 1. All markers were decreased on day 1 and started to increase again and reached peak levels $(53.1 \pm 18.0 \mathrm{pg} / \mathrm{ml}, 6.43 \pm 2.50 \mathrm{pg} / \mathrm{ml}, 66.3 \pm 27.6 \mathrm{ng} / \mathrm{l}$, $6.44 \pm 2.40 \mathrm{ng} / \mathrm{l}$, and $1885 \pm 921 \mathrm{pg} / \mathrm{ml}$, respectively) on day 5 after saline injection. IL-1 $\beta$, IL-6, IL-10, TNF- $\alpha$, and PG-E $\mathrm{E}_{2}$ concentrations in the synovial fluid from the PRP group are presented in Table 2. In the PRP group, 
Tab. 1. The changes in parameter levels in saline-injected group (mean \pm SE). IL-1 $\beta$, IL-6, IL-10, TNF- $\alpha$, and PG-E ${ }_{2}$ concentrations $(P>0.05)$ showed no statistically significant changes in the control groups, although fluctuations in all parameters were observed

\begin{tabular}{|c|c|c|c|c|c|c|c|c|c|}
\hline \multirow{2}{*}{ Parameters } & \multicolumn{9}{|c|}{ Days } \\
\hline & 0 & 1 & 3 & 5 & 7 & 14 & 30 & 60 & 90 \\
\hline IL-1及 (pg/ml) & $83.4 \pm 33.2$ & $38.4 \pm 13.9$ & $41.1 \pm 7.86$ & $53.1 \pm 18.0$ & $51.0 \pm 5.59$ & $39.4 \pm 13.9$ & $27.7 \pm 4.10$ & $19.4 \pm 3.19$ & $20.38 \pm 3.18$ \\
\hline IL-6 (pg/ml) & $10.3 \pm 4.62$ & $4.12 \pm 2.16$ & $4.06 \pm 0.99$ & $6.43 \pm 2.50$ & $5.35 \pm 0.98$ & $4.03 \pm 1.48$ & $2.64 \pm 0.55$ & $2.13 \pm 0.62$ & $2.32 \pm 0.66$ \\
\hline IL-10 (ng/l) & $87.8 \pm 41.3$ & $39.2 \pm 17.2$ & $38.2 \pm 13.8$ & $66.3 \pm 27.6$ & $50.9 \pm 7.77$ & $28.3 \pm 15$ & $19.0 \pm 4.40$ & $8.72 \pm 5.62$ & $20.8 \pm 5.49$ \\
\hline TNF- $\alpha(\mathrm{pg} / \mathrm{ml})$ & $10.8 \pm 4.57$ & $4.89 \pm 2.09$ & $4.61 \pm 1.03$ & $6.44 \pm 2.40$ & $5.26 \pm 0.49$ & $5.18 \pm 2.02$ & $3.05 \pm 0.57$ & $2.27 \pm 0.56$ & $2.24 \pm 0.69$ \\
\hline$P G-E_{2}(p g / m l)$ & $14.7 \pm 6.54$ & $54.0 \pm 32.9$ & $102 \pm 45.8$ & $1885 \pm 921$ & $17.9 \pm 10.05$ & $94.4 \pm 51.1$ & $32.7 \pm 20.0$ & $45.9 \pm 15.4$ & $42.3 \pm 24.5$ \\
\hline
\end{tabular}

Explanation: no statistical difference was determined in the same line $(\mathrm{P}>0.05)$

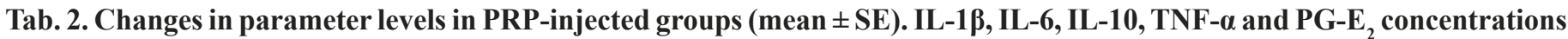
$(P>0.05)$ showed no statistically significant changes in the PRP groups, although fluctuations in all parameters were observed

\begin{tabular}{|c|c|c|c|c|c|c|c|c|c|}
\hline \multirow{2}{*}{ Parameters } & \multicolumn{9}{|c|}{ Days } \\
\hline & 0 & 1 & 3 & 5 & 7 & 14 & 30 & 60 & 90 \\
\hline IL-1及 (pg/ml) & $16.7 \pm 4.02$ & $7.39 \pm 2.04$ & $12.3 \pm 2.74$ & $13.3 \pm 2.70$ & $15.5 \pm 4.68$ & $19.2 \pm 3.80$ & $15.9 \pm 4.48$ & $9.09 \pm 2.90$ & $13.4 \pm 3.67$ \\
\hline IL-6 (pg/ml) & $3.16 \pm 0.48$ & $2.42 \pm 0.40$ & $3.16 \pm 0.47$ & $3.58 \pm 0.70$ & $3.71 \pm 0.80$ & $5.13 \pm 1.04$ & $3.13 \pm 0.62$ & $3.31 \pm 0.77$ & $3.63 \pm 0.81$ \\
\hline IL-10 (ng/l) & $37.6 \pm 4.28$ & $32.1 \pm 5.47$ & $39.0 \pm 5.69$ & $40.5 \pm 7.30$ & $48.0 \pm 9.16$ & $40.0 \pm 7.97$ & $27.2 \pm 4.01$ & $32.0 \pm 4.98$ & $39.5 \pm 9.36$ \\
\hline TNF- $\alpha(p g / m l)$ & $2.30 \pm 0.46$ & $1.28 \pm 0.18$ & $1.66 \pm 0.23$ & $2.17 \pm 0.44$ & $2.21 \pm 0.30$ & $2.96 \pm 0.43$ & $2.61 \pm 0.55$ & $3.23 \pm 0.72$ & $2.57 \pm 0.49$ \\
\hline$P G-E_{2}(p g / m l)$ & $4.37 \pm 0.39$ & $7.94 \pm 1.60$ & $12.2 \pm 2.89$ & $8.70 \pm 1.68$ & $7.00 \pm 1.37$ & $4.16 \pm 0.35$ & $14.0 \pm 3.65$ & $4.17 \pm 0.31$ & $7.11 \pm 1.09$ \\
\hline
\end{tabular}

Explanation: as in Tab. 1.

IL-1 $\beta$ and IL-6 levels were highest $(19.2 \pm 3.80 \mathrm{pg} / \mathrm{ml}$ and $5.13 \pm 1.04 \mathrm{pg} / \mathrm{ml}$, respectively) on day 14 . Peak levels were observed on day 7 for IL-10 $(48.0 \pm 9.16$ $n g / 1)$ and on day 60 for TNF- $\alpha(3.23 \pm 0.72)$ after PRP treatment. $\mathrm{PG}-\mathrm{E}_{2}$ concentrations remained low (ranging from $4.16 \pm 0.35$ to $14.0 \pm 3.65 \mathrm{pg} / \mathrm{ml}$ ) for the duration of the study and were highest on day $30(14.0 \pm 3.65$ $\mathrm{pg} / \mathrm{ml}$ ) in the PRP group.

PRP may have a favorable effect on OA in dogs. The present study hypothesized that a single intra-articular injection of autologous platelet concentrate resulted in significant clinical improvements within 12 weeks in dogs with OA involving a single joint administration, as Fahie et al. (9) previously described.

Several inflammatory mediators (IL-1 $\beta$, IL-6, TNF- $\alpha$, and $\left.\mathrm{PG}-\mathrm{E}_{2}\right)$ increase in $\mathrm{OA}(7,12,14)$. We believe that arthrocentesis and measurement of potential disease markers can provide more objective information regarding the effects of treatment procedures. For this reason, the inflammatory mediators were determined in the joint fluid after treatment. In the present study, all of the markers were determined at specific quantities in the evaluation of the joint fluid in all osteoarthritic cases in both groups. IL-1, as a major inflammatory and catabolic cytokine in the pathophysiology of OA, represents one of the possible treatment targets. IL-1 $\beta$ levels are significantly increased in osteoarthritic synovial fluid obtained in both early and late OA (14). Moreover, the prevalence of IL-1 $\beta$ in the pathogenesis of OA has been reported (15). In the present study, the lowest concentrations of IL-1 $\beta(\mathrm{pg} / \mathrm{ml}$ ) occurred earlier in the PRP group (day 1 at $7.39 \pm 2.04$ ) compared to the saline group (day 60 at $19.4 \pm 3.19$ ), but this difference was not statistically significant. All inflammatory mediators were detected at different rates in all dogs with OA in both groups because the samples were obtained at different stages of OA, rather than at standardized times, as in an experimental study by Venn et al. (20).

In this study, it was determined that all inflammatory mediators fluctuated in the synovial fluid in the control and PRP groups. This fluctuation may have contributed to the persisting inflammatory symptoms, but the TNF- $\alpha$ ratio was slightly decreased during the first week after PRP administration. In addition, PRP contains a large amount of stem cells (16). Previous studies have demonstrated a correlation between a TNF- $\alpha$ increase in joint fluid and the OA pain score (17). The decreased synthesis of TNF- $\alpha$, which is not statistically significant even in a short period of time, explains one of the mechanisms of PRP in joint tissues and clinical practice (17). It has been noted that the suppression of TNF- $\alpha$ synthesis reduces pain. It is responsible for the removal of inflammatory debris, shock absorption, lubrication, and the inflammatory conditioning of catabolic cytokines (19). IL-6 has been demonstrated to be elevated in OA joint fluid, particularly in acute conditions $(18,19)$. In the PRP group, IL-6 concentration slightly decreased within the first days after injection, but increases were observed at the end of the $14^{\text {th }}$ day. IL- 6 reached peak levels on day 14 after PRP administration and on day 7 after saline injection. IL-6 concentration has been demonstrated to decrease in late OA (18). Because, IL-6 has a pro-inflammatory effect (21).

In this study, there were also no statistically significant increases in IL-10 levels in the control and PRP groups. IL-10 synthesis plays a role in the suppression of IL-1 $\beta$ and TNF- $\alpha$ synthesis during disease process. In the present study, we observed the lowest IL-10 level 
on day $60(8.72 \pm 5.62 \mathrm{ng} / \mathrm{ml})$ in the control group, whereas the minimum level of IL-10 in the PRP group was $27.2 \pm 4.01 \mathrm{ng} / \mathrm{ml}$. It was found that TNF- $\alpha$ and PG-E 2 activity decreased in 6 out of 14 (42.8\%) dogs when evaluated individually in the PRP group. In particular, it seems that PRP should be administered more frequently at specific intervals in order to prevent a slight rise in TNF- $\alpha$ after the fifth day, since TNF- $\alpha$ plays an important role in the onset of inflammatory reactions in the joint. However, the inflammatory mediators were unexpected high in the saline group compared to the PRP group. This is because the control group received an injection of 3-5 $\mathrm{ml}$ of saline solution, which caused the inflammatory mediators to be diluted in the synovial fluid. For this reason, a significant reductions in markers occurred from day 1 post-surgery in the control group. However, this decrease lasted for five days, and then the parameters increased again. Therefore, dilution effects should always be considered (18). In the present study, PG-E levels increased markedly in the control group (from $14.7 \pm 6.54$ to $1885 \pm 921$ ), although they fluctuated in both groups. This finding suggests that PRP treatment can prevent increasing $\mathrm{PG}-\mathrm{E}_{2}$ levels, but not significantly.

Dogs receiving PRP improved clinically, but the radiographic scores assigned at week 12 were the same as the scores assigned at pre-treatment. The ultrasonography examination confirmed a decrease in effusion in the joint. Although our study indicates that lameness in the saline-injected group abated at day 5, lameness increased again by day 21 . This initial improvement may have resulted from the dilution of inflammatory mediators by the saline injection.

It has been suggested that the administration of high concentrations of platelets would lead to high concentrations of the growth factors, and thus have the potential to augment or stimulate the healing process that normally occurs after injury (18). Platelet-rich plasma concentrate, when administered intra-articularly, may also have the potential to slow the progression of OA by stimulating cartilage anabolism $(18,20)$. In addition to growth factors, PRP also contains plasma proteins which act as mesenchymal cell adhesion molecules (19). After injection into the damaged tissue, the platelets in PRP begin active secretion of growth factors within $10 \mathrm{~min}$, with $>95 \%$ of the total amount being released within the first hour (9). The secretion of growth factors occurs mainly within the first hour of injection; the platelets remain viable for 7 days and continue to release growth factors. It has been suggested that a single injection of PRP into the damaged tissue might be sufficient in most cases (9).

PRP injection seems to be an effective and safe method for treating dogs with $\mathrm{OA}$; in vivo data and clinical results are encouraging. The entire procedure, from initial sedation to the completion of intra-articular injection, takes about 35-40 min. PRP could be more beneficial for early cases of mild and moderate OA. Despite some variable results on inflammatory parameters, clinical improvement was recorded in the PRP-treated group. that PRP could have an anti-inflammatory effect.

\section{References}

1. Aragon C. L., Hofmeister E. H., Budsberg S. C.: Systematic review of clinical trials of treatments for osteoarthritis in dogs. JAVMA 2007, 230, 514-521.

2. Arican M., Şimşek A., Parlak K., Atli K., Sönmez G.: Matrix metalloproteinases 2 and 9 activity after intra-articular injection of autologous platelet-rich plasma for the treatment of osteoarthritis in dogs. Acta Vet. Brno 2018, 87, 127-135.

3. Arican M., Şimşek A., Parlak K., Atli K., Sönmez G.: The effect of matrix metalloproteinases 2 and 9, TNF- $\alpha$ and PGE-2 activity after intra-articular injection of an autologous plasma rich platelets for the treatment of osteoarthritis in dogs. 15-19 October, The Southern European Veterinary Conference. 2015 Barcelona, Spain (Best Awards).

4. Brenner S. S., Klotz U., Alscher D. M., Mais A., Lauer G., Schweer H., Seyberth H. W., Fritz P., Bierbach U.: Osteoarthritis of the knee: clinical assessments and inflammatory markers. Osteoarthr. Cartil. 2004, 12, 469-475.

5. Brown D. C.: What can we learn from osteoarthritis pain in companion animals? Clin. Exp. Rheumatol. 2017, 107, 53-58.

6. Brown D. C., Boston R. C., Coyne J. C.: Ability of the canine brief pain inventory to detect response to treatment in dogs with osteoarthritis. JAVMA 2008, 233, 1278-1283.

7.Dayer J. M.: The Process of identifying and understanding cytokines: from basic studies to treating rheumatic diseases. Best Pract. Res. Clin. Rheumatol. 2004, 18, 31-45.

8.Dohan D. M., Bielecki T., Del Corso M., Inchingolo F., Sammatino G.: Shedding light in the controversial terminology for platelet-rich products: Platelet-Rich Plasma (PRP), Platelet-Rich Fibrin (PRF), Platelet-Leukocyte Gel (PLG), Preparation Rich in Growth Factors (PRGF), classification and commercialism. J. Biomed. Mater. Res. 2010, 95, 1280-1282.

9. Fahie M. A., Ortolano G. A., Gurecio V., Schaffer J. A., Johnston G., Au J., Hettlich B. A., Philips T., Allen M. J., Bertone A. L.: A randomized controlled trial of the efficacy of autologous plasma rich platelet therapy for the treatment of osteoarthritis in dogs. JAVMA 2013, 243, 1291-1297.

10. Fortier L. A., Travis A. J.: Stem cells in veterinary medicine. Stem Cell Res. Ther. 2011, 1, 9 .

11. Franklin S. P., Garner B. C., CookJ. L.: Characteristics of canine platelet-rich plasma prepared with five commercially available systems. AJVR 2015, 76, 822-827.

12. Hegemann N., Wondimu A., Kohn B., Brunnberg L., Schmidt M. F.: Cytokine profile in canine immune-mediated polyarthritis and osteoarthritis. Vet. Comp. Orthopaed. 2005, 18, 67-72.

13. Hudson J. T., Slater M. R., Taylor L., Scott M., Kerwin S. C.: Assessing repeatability and validity of a visual analogue scale questionnaire for use in assessing pain and lameness in dogs. AJVR 2004, 65, 1634-1643.

14. Joosten L. A., Helsen M. M., Saxne T., Heinegard D., Van Den Berg W. B.: IL-1 alpha beta blockade prevents cartilage and bone destruction in murine type ii collagen-induced arthritis, whereas TNF alpha blockade only ameliorates joint inflammation. J. Immunol. 1999, 163, 5049-5055.

15. Kapoor M., Martel-Pelletier J., Lajeunesse D., Pelletier J. P., Fahmi H.: Role of proinflammatory cytokines in the pathophysiology of osteoarthritis. Nat. Rev. Rheumatol. 2011, 7, 33-42.

16. Leong D. K. C., Benedict B. C. T., Chew K. T. L.: Autologous Growth Factors: A biological treatment in sports medicine. Proceedings of Singapore Helatcare 2012, 19, 229-236.

17. Ohtori S., Takahashi K., Moriya H., Myers R. R.: TNF-Alpha and TNF-Alpha Receptor Type 1 upregulation in glia and neurons after peripheral nerve injury: Studies in murine drg and spinal cord. Spine. 2004, 29, 1082-1088.

18. Sohn D., Sokolove J., Sharpe O., Erhart J., Chandra P., Lahey L., Lindstrom T. M., Hwang I., Boyer K. A., Andriacchi T. P., Robinson W. H.: Plasma proteins present in osteoarthritic synovial fluid can stimulate cytokine production via toll-like receptor 4. Arthritis Res. Ther. 2012, 14, R7.

19. Stief M., GottschalkJ., Ionita J. C., Einspanier A., Oechtering G., Böttcher P.: Concentration of platelets and growth factors in canine autologous conditioned plasma. Vet. Comp. Orthopaed. 2011, 24, 122-125.

20. Venn G., Nietfeld J. J., Duits A. J., Brennan E., Arner M., Covington M. E., Billingham T. E.: Elevated synovial fluid levels of interleukin-6 and tumor necrosis factor associated with early experimental canine osteoarthritis. Arthritis Rheumatol. 2005, 36, 819-826.

21. Xing Z., Gauldie J., Cox G., Baumann H., Jordana M., Lei X. F., Achong M. K. IL-6 is an anti-inflammatory cytokine required for controlling local or systemic acute inflammatory responses. J. Clin. Invest. 1998, 101, 311-320.

Corresponding author: Dr. Arican Mustafa, Department of Surgery, Faculty of Veterinary Medicine, The University of Selcuk, Konya, Turkey; e-mail: marican@selcuk.edu.tr 